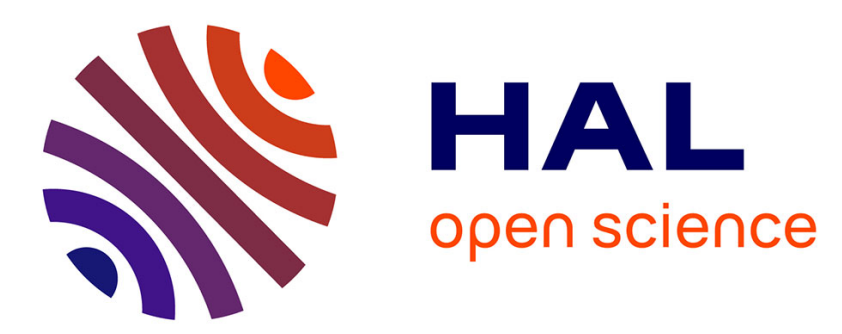

\title{
Time-resolved, optically detected NMR of fluids at high magnetic field
}

\author{
Daniela Pagliero, Wei Dong, Dimitris Sakellariou, Carlos A. Meriles
}

\section{To cite this version:}

Daniela Pagliero, Wei Dong, Dimitris Sakellariou, Carlos A. Meriles. Time-resolved, optically detected NMR of fluids at high magnetic field. Journal of Chemical Physics, 2010, 133, pp.154505. $10.1063 / 1.3502484$. cea-00854029

\section{HAL Id: cea-00854029 https://hal-cea.archives-ouvertes.fr/cea-00854029}

Submitted on 24 Sep 2019

HAL is a multi-disciplinary open access archive for the deposit and dissemination of scientific research documents, whether they are published or not. The documents may come from teaching and research institutions in France or abroad, or from public or private research centers.
L'archive ouverte pluridisciplinaire $\mathbf{H A L}$, est destinée au dépôt et à la diffusion de documents scientifiques de niveau recherche, publiés ou non, émanant des établissements d'enseignement et de recherche français ou étrangers, des laboratoires publics ou privés. 


\section{Time-resolved, optically detected NMR of fluids at high magnetic field}

Cite as: J. Chem. Phys. 133, 154505 (2010); https://doi.org/10.1063/1.3502484

Submitted: 29 May 2010 . Accepted: 22 September 2010 . Published Online: 18 October 2010

Daniela Pagliero, Wei Dong, Dimitris Sakellariou, and Carlos A. Meriles

\section{ARTICLES YOU MAY BE INTERESTED IN}

Angle sensing with ferromagnetic nanowire arrays

Applied Physics Letters 104, 032403 (2014); https://doi.org/10.1063/1.4862924

Reduced electron temperature in a magnetized inductively-coupled plasma with internal coil Physics of Plasmas 20, 103504 (2013); https://doi.org/10.1063/1.4825135

Electron and hole spin cooling efficiency in InAs quantum dots: The role of nuclear field Applied Physics Letters 96, 172108 (2010); https://doi.org/10.1063/1.3394010

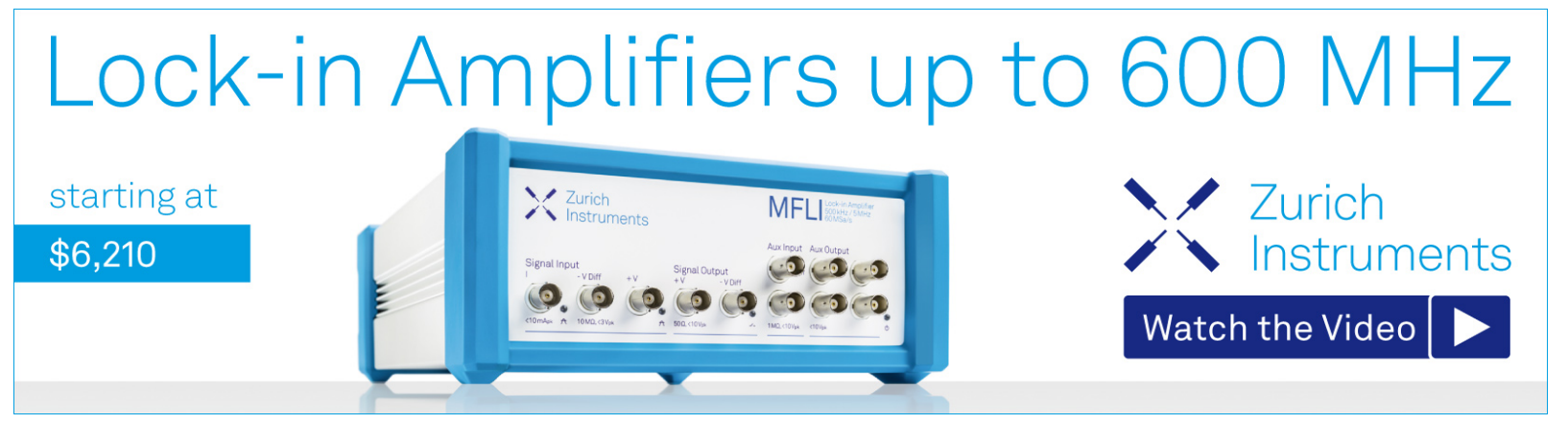

J. Chem. Phys. 133, 154505 (2010); https://doi.org/10.1063/1.3502484 


\title{
Time-resolved, optically detected NMR of fluids at high magnetic field
}

\author{
Daniela Pagliero, ${ }^{1}$ Wei Dong, ${ }^{1}$ Dimitris Sakellariou, ${ }^{2}$ and Carlos A. Meriles ${ }^{1, a)}$ \\ ${ }^{1}$ Department of Physics, The City College of New York, CUNY, 138th Street and Convent Avenue, \\ New York, New York 10031, USA \\ ${ }^{2}$ Laboratoire de Structure et Dynamique par Résonance Magnétique, Service Interdisciplinaire sur les \\ Systèmes Moléculaires et les Matériaux, DSM/IRAMIS/SIS2M, CEA Saclay, Gif-sur-Yvette 91191, France
}

(Received 29 May 2010; accepted 22 September 2010; published online 18 October 2010)

\begin{abstract}
We report on the use of optical Faraday rotation to monitor the nuclear-spin signal in a set of model ${ }^{19} \mathrm{~F}$ - and ${ }^{1} \mathrm{H}$-rich fluids. Our approach integrates optical detection with high-field, pulsed NMR so as to record the time-resolved evolution of nuclear-spins after $\mathrm{rf}$ excitation. Comparison of chemical-shift-resolved resonances allows us to set order-of-magnitude constrains on the relative amplitudes of hyperfine coupling constants for different bonding geometries. When evaluated against coil induction, the present detection modality suffers from poorer sensitivity, but improvement could be attained via multipass schemes. Because illumination is off-resonant i.e., the medium is optically transparent, this methodology could find extensions in a broad class of fluids and soft condensed matter systems. (C) 2010 American Institute of Physics. [doi:10.1063/1.3502484]
\end{abstract}

\section{INTRODUCTION}

While the use of optical schemes to polarize and detect nuclear-spins is well known, applications to date have been mostly restricted to atomic vapors and select condensed matter systems. ${ }^{1,2}$ Early extensions to fluids focused on the response of resonance frequencies to illumination with circularly polarized light far detuned from optical transitions, a strategy thought to enable new forms of "light-assisted" NMR spectroscopy. ${ }^{3}$ Although subsequent work showed that light-induced shifts of chemical-shift resolved resonances are too small $\left(\sim 10^{-5} \mathrm{~Hz}\right)$ to be observed in practice, ${ }^{4,5}$ the converse effect, i.e., changes in the plane of polarization of a light beam due to nuclear-spin magnetization, has been recently exploited to demonstrate optical detection of proton magnetic resonance in water. ${ }^{6}$ To attain a level of signal akin to the sensitivity of state-of-the-art polarimeters, the experiment of Ref. 6 relied on a $50 \mathrm{~cm}$ long water sample prepolarized to the equivalent of $1.5 \mathrm{~T}$. With the fluid continuously circulating from a high- to a low-field magnet $(\sim 5 \mathrm{G})$ detection was carried out at $21 \mathrm{kHz}$ using a cw "spin-locking" protocol. Here, we extend these results with observations of ${ }^{19} \mathrm{~F}$ - and ${ }^{1} \mathrm{H}$-induced optical Faraday rotation (OFR) in model liquid samples at high magnetic field. Our approach articulates OFR with detection schemes well known in modern nuclear magnetic resonance (NMR), a feature that allows us to implement time-resolved, multipulse sequences and measure light-encoded chemical-shift-resolved spectra.

To derive the equations describing the effect of nuclearspin on the polarization of a light beam, we start with the traditional representation of OFR in terms of the magneticfield-induced difference between the indices of refraction $n_{+}$ and $n_{-}$for left- and right-circularly polarized lights. ${ }^{7,8} \mathrm{~A}$ straightforward calculation shows that the rotation of the

\footnotetext{
${ }^{\text {a) }}$ Author to whom correspondence should be addressed. Electronic mail: cmeriles@sci.ccny.cuny.edu.
}

plane of polarization of a linearly polarized beam of wavelength $\lambda$ upon traversing a sample of length $L$ is given by $\theta^{(F)}=(\pi / \lambda)\left(n_{+}-n_{-}\right) L$. For a dielectric medium with molecular number density $N$ and assuming that the beam propagates along the $x$-axis, Maxwell equations allow us to recast $\theta^{(F)}$ in terms of the antisymmetric components $\alpha_{i j}^{\prime}$ of the electric polarizability tensor $\overleftrightarrow{\alpha}$. One finds ${ }^{7}$

$$
\theta^{(F)}=\frac{1}{2} \mu_{0} c \omega L N \alpha_{y z}^{\prime},
$$

where $\mu_{0}$ denotes the vacuum magnetic permeability, $c$ is the speed of light, and $\omega$ the frequency. For a diamagnetic material, first-order perturbation theory in the presence of a magnetic field $B_{1}$ along $x$ yields the dynamic polarizability, ${ }^{7}$

$$
\alpha_{y z}^{\prime}(\mathbf{B})=\alpha_{y z}^{\prime}+\alpha_{y z, x}^{(D)} B_{1},
$$

where

$$
\alpha_{y z, x}^{\prime(D)} \sim K \omega \sum_{|e\rangle \notin|g\rangle} \frac{\omega_{e g}}{\left(\omega_{e g}^{2}-\omega^{2}\right)^{2}}\left(m_{x}^{e e}-m_{x}^{g g}\right) .
$$

In Eq. (3), the sum extends over all dipole-allowed transitions of frequencies $\omega_{p q} ;|g\rangle,|e\rangle$ denote molecular ground and excited states, respectively, $m_{x}^{r s} \equiv\left\langle r\left|m_{x}\right| s\right\rangle$ indicates the $x$-component of the magnetic dipolar moment, and $K$ is a proportionality constant. The product $V=(1 / 2) \mu_{0} c \omega N \alpha_{y z, x}^{\prime(D)}$ is called the "Verdet constant" of the material under consideration and measures the rotation per unit magnetic field and per unit length. Although $B_{1}$ is typically assumed static, formula (2) is valid for time-dependent fields $B_{1}(t)$ $=B_{1}^{0} \cos \left(2 \pi \nu_{1} t\right)$ so long as the inverse of the frequency $\nu_{1}$ is greater than a time $\tau_{e}$ characterizing electronic relaxation (typically $\sim 1$ ns or shorter). Further, we note that Faraday rotation is insensitive to components of the magnetic field perpendicular to the direction of propagation to leading order. In the context of our experimental apparatus (where, keeping with NMR nomenclature, the large Zeeman field $\left(B_{0}\right)$ is taken along the $z$-axis), the latter will prove a crude, 

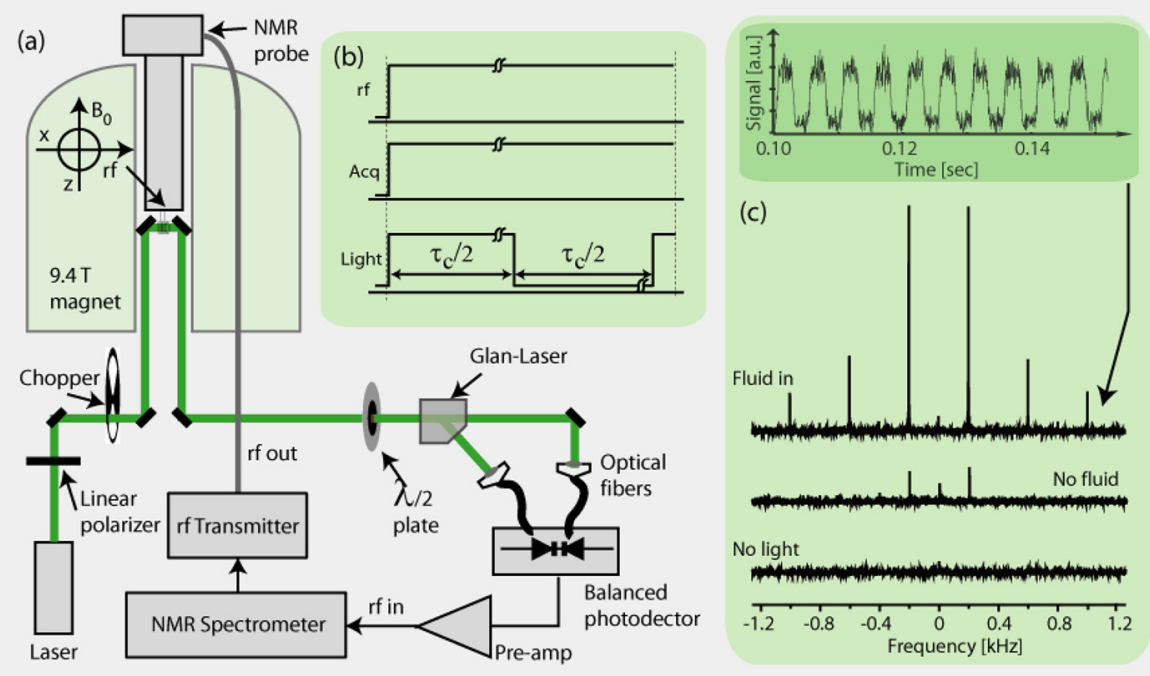

FIG. 1. Detection of regular OFR in fluids at high magnetic field. (a) Experimental setup. (b) Pulse sequence. To more clearly identify light-encoded signal, the laser beam is chopped at a frequency $1 / \tau_{\mathrm{c}}$. Acquisition and rf excitation start in synchrony with the positive edge of the chopper digital monitor output. (c) Fourier transforms of the observed OFR signal after $501 \mathrm{~s}$ acquisitions. Peaks at multiples of the light modulation frequency are observed when the sample fluid $\left(\mathrm{C}_{6} \mathrm{~F}_{14}\right)$ fills the container (upper trace). Background peaks remaining after the fluid is removed (medium trace) originate from the glass windows at each end of our cylindrical container. With (or without) sample fluid, no background signal is present if the laser is blocked. The absence of the central peak at the carrier frequency $(370 \mathrm{MHz})$ results from dc offset correction during signal processing. In all cases, the rf field amplitude was $40 \mu \mathrm{T}$, and the beam intensity at the photodetectors was $1.5 \mathrm{~mW}$.

yet useful, approximation. Later in this paper we will return to this issue.

Similar to other optical schemes, the use of Faraday rotation to probe nuclear-spins exploits the hyperfine interaction with electrons, the strength of which depends on the system under consideration. In organic materials-where valence electrons are best described by wave functions with nodes at the nuclear coordinates-hyperfine couplings are typically weak. Nonetheless, dipolar and residual "contact" terms amount to a nonvanishing electron-nuclear coupling, formally represented through the hyperfine Hamiltonian $H_{\mathrm{hf}} \cdot{ }^{9}$ Following a reasoning similar to that of formulas (2) and (3), one can write the change of the molecular susceptibility due to the nuclear magnetization as ${ }^{10}$

$$
\Delta \alpha_{y z}^{\prime(N)}=\alpha_{y z, x}^{\prime(N)}\left\langle I_{x}\right\rangle
$$

where $\alpha_{y z, x}^{\prime(N)}$ is obtained from formula (3) with the correspondence $B_{x} m_{x}^{e g} \rightarrow H_{\mathrm{hf}}^{e g}=a^{e g}\left\langle I_{x}\right\rangle ; a^{e g}$ denotes the set of hyperfine constants and $\left\langle I_{x}\right\rangle$ is the nuclear polarization along the direction of propagation of the beam. For a system of molecules in equilibrium, we write the contribution of the nuclear magnetization to the overall Faraday rotation,

$$
\theta^{(N)}=\frac{1}{2} \mu_{0} c \omega L N \alpha_{y z, x}^{\prime(N)}\left\langle I_{x}\right\rangle \sim \theta^{(F)} \frac{B_{N}}{B_{1}^{0}} .
$$

It follows that the effect of a net nuclear-spin magnetization on the electronic system amounts to the application of an external nuclear magnetic field $B_{N} \sim\left(a_{H} /\left(g^{*} \mu_{B}\right)\right)\left\langle I_{x}\right\rangle$ along the $x$-axis, where $a_{H}$ is a magnitude representative of the hyperfine constant matrix, $g^{*}$ denotes the Landé factor, and $\mu_{B}$ is the Bohr magneton. We thus surmise that precession of the nuclear magnetization following a resonant $\mathrm{rf}$ excitation leads to a Faraday rotation signal precisely at the nuclear Larmor frequency. In Sec. II, we take advantage of this effect to probe nuclear-spins optically using typical pulsed-NMR techniques.

\section{RESULTS}

A sketch of our experimental setup is shown in Fig. 1: Linearly polarized light from a $532 \mathrm{~nm}$ laser propagates in and out of a $\sim 1 \mathrm{~cm}^{3}$ sample fluid within the bore of a $9.4 \mathrm{~T}$ NMR magnet. The sample container-shaped in the form of a $\sim 2 \mathrm{~cm}$ long hollow cylinder with optical windows in each end-is oriented so that the beam travels perpendicular to the direction of the external dc field $B_{0} \hat{\mathbf{z}}$. An eight-turn solenoid wound on the outer surface of the container, and part of a homemade NMR probe, produces a magnetic field $B_{1} \hat{\mathbf{x}}$ at the ${ }^{19} \mathrm{~F}$ or ${ }^{1} \mathrm{H}$ resonance frequency $(\sim 376$ and $400 \mathrm{MHz}$, respectively). Detection of Faraday rotation is carried out with a $\lambda / 2$-plate, a Glan-laser polarizer, and a balanced, broadband photoreceiver; the resulting signal is fed into a commercial NMR spectrometer for final demodulation and processing.

We start in Fig. 1 with initial observations of regular Faraday rotation as described by formula (1) in a sample of perfluorohexane $\left(\mathrm{C}_{6} \mathrm{~F}_{14}\right)$. Continuous radio-frequency ( $\mathrm{rf}$ ) excitation at a preset frequency (within the range 1$400 \mathrm{MHz}$ ) creates an oscillating $B_{1}$ field over the sample volume of amplitude $40 \mu \mathrm{T}$. Synchronous mechanical chopping of the beam amplitude at a frequency $1 / \tau_{c}=200 \mathrm{~Hz}$ was introduced to separate light-encoded signal from unintentional pickup. ${ }^{11}$ The upper trace in Fig. 1(c) shows the Fourier transform of the OFR signal after fifty $1 \mathrm{~s}$ acquisitions at $370 \mathrm{MHz}$. The spectrum displays the expected satellite pattern due to square modulation of the light amplitude [see insert to Fig. 1(c)]; note that the signal virtually disappears if the fluid is removed or if one blocks the beam before 


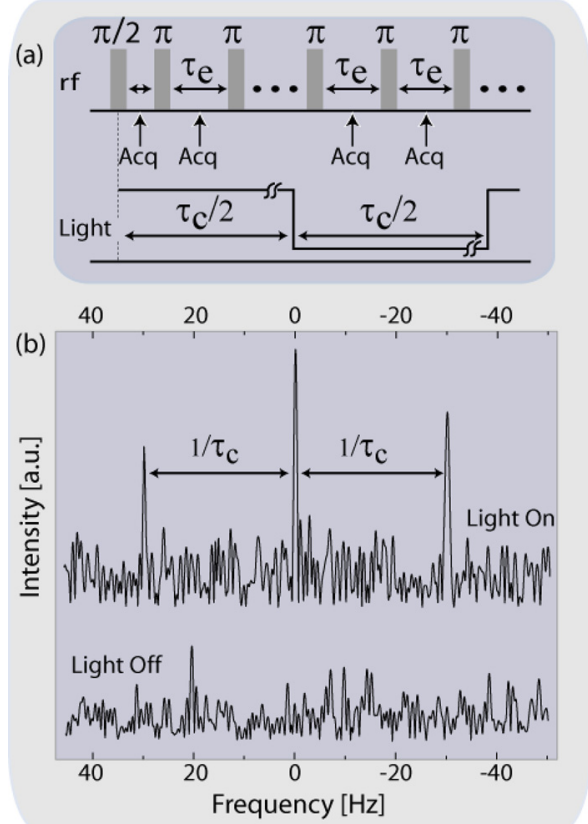

FIG. 2. Detection of nuclear-spin-induced OFR in $\mathrm{C}_{6} \mathrm{H}_{14}$. (a) CPMG train and synchronous light modulation. The separation between inversion pulses was $8 \mathrm{~ms}$ and the total number of echoes was 200. (b) ${ }^{19} \mathrm{~F}$ ODNMR signal in $\mathrm{C}_{6} \mathrm{~F}_{14}$ after $5 \times 10^{4}$ scans. Notice the satellites at the light modulation frequency $1 / \tau_{\mathrm{c}}=30 \mathrm{~Hz}$.

the photodetectors. To calibrate and test our setup, we conducted extensive measurements as a function of light intensity, rf amplitude and frequency, and number of scans. ${ }^{11}$ Our system proved robust to time fluctuations, with a signal-tonoise ratio (SNR) growing, as expected, with the square root of the number of repetitions (up to $5 \times 10^{4}$ ). The SNR increases linearly with the laser beam intensity and with the rf amplitude.

To induce and observe nuclear-spin OFR, we implemented a CPMG sequence. ${ }^{12}$ Again, a synchronous chopper was used to modulate the beam amplitude at a frequency $1 / \tau_{c}=30 \mathrm{~Hz}$. When compared with the simpler excitationacquisition protocol typical of pulsed NMR, the use of a CPMG train allows us to sustain the nuclear-spin coherence for longer periods of time, thus leading to an improved SNR for the same number of repeats. The plot in Fig. 2 shows the Fourier transform of the observed "free induction decay" assembled as the collection of single-point stroboscopic acquisitions at the midpoint of the interpulse intervals $\tau_{\mathrm{e}}$; the total number of scans was $5 \times 10^{4}$. In addition to the expected central peak (resulting from the overall exponential decay of the nuclear-spin-echo maxima), the "laser-on" spectrum shows two symmetric satellites at frequencies $\omega_{c}^{ \pm}= \pm 1 / \tau_{c}$, an unambiguous ${ }^{11}$ indication of light-encoded signal. We stress that unintended rf pickup is negligible, as shown by the fact that all resonance peaks - including the central peak at the carrier frequency-disappear if the beam, still traversing the sample volume, is diverted from the photodetectors.

While the presence of resonance satellites is crucial to identify the origin of the observed signal (optical versus rf pickup), laser chopping reduces the resulting SNR and the appearance of satellites considerably complicates extensions to information-richer schemes. In Fig. 3, we exploit the ab-

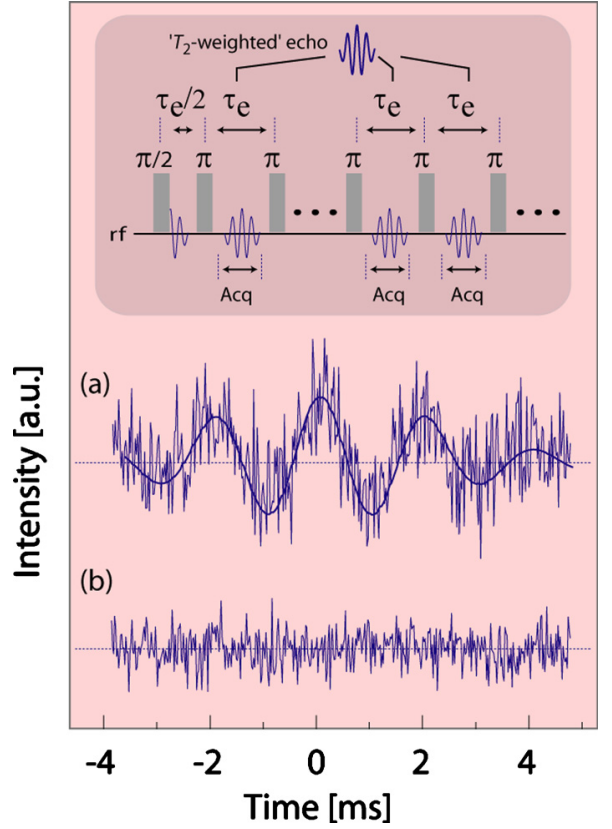

FIG. 3. (Insert) Schematics of the detection protocol. After excitation, a train of inversion pulses generates a corresponding train of nuclear-spin echoes. Unlike the experiment of Fig. 2, detection is carried out during the duration of each interpulse interval $\tau_{\mathrm{e}}$. (Main) (a) OFR-detected ${ }^{19} \mathrm{~F}$ " $T_{2}$-weighted" echo after coadding individual echoes in the train (see text). The "smooth" solid line corresponds to the inductively detected $T_{2}$-weighted echo, superimposed for reference. (b) Same as in (a) but when the light is off. An identical (null) result is obtained when the beam, while illuminating the photodetector, is diverted to bypass the sample. In (a) and (b) the sample is hexafluorobenzene $\left(\mathrm{C}_{6} \mathrm{~F}_{6}\right)$ and the total number of acquisitions is $8 \times 10^{3}$.

sence of background signal to optically detect the timeresolved ${ }^{19} \mathrm{~F}$ echo in a sample of hexafluorobenzene $\left(\mathrm{C}_{6} \mathrm{~F}_{6}\right)$. We still use a CPMG train to optimize detection sensitivity, but in this case we extend the acquisition window to the interpulse interval. During processing, each spin echo in the string is extracted and coadded to reveal a " $T_{2}$-weighted" signal. Traces (a) and (b) in the main figure panel summarize our observations: Under illumination, we observe a clear echo signal exhibiting a $\sim 500 \mathrm{~Hz}$ beating (the expected consequence of shifting that much the spectrometer carrier frequency, $\sim 376 \mathrm{MHz}$, from the ${ }^{19} \mathrm{~F}$ NMR resonance in $\mathrm{C}_{6} \mathrm{~F}_{6}$ ). We find excellent correspondence with the inductively detected, $T_{2}$-weighted echo, superimposed for reference. Further confirming our observations of Fig. 2, we find no signal when the light beam is off and/or when the beam path is altered to illuminate the photodetector while bypassing the sample.

Capitalizing on the strong $B_{0}$ field, one can extend these observations to carry out a chemical-shift resolved characterization of the sample. The latter is presented in Fig. 4 for an admixture of hexafluorobenzene and perfluorohexane $\left[\mathrm{CF}_{3}-\left(\mathrm{CF}_{2}\right)_{4}-\mathrm{CF}_{3}\right.$ or $\mathrm{C}_{6} \mathrm{~F}_{14}$ for short]. Given our relatively long inversion pulses $(\sim 50 \mu \mathrm{s})$ and the large frequency difference between the $\mathrm{CF}_{3}$ (left) and aromatic (right) resonances $(\sim 30 \mathrm{kHz})$, two separate observations at different carrier frequencies (dashed lines) were necessary. Figures 4(a) and 4(b) show the results for inductive and optical detection, respectively, after Fourier transform (magnitude 


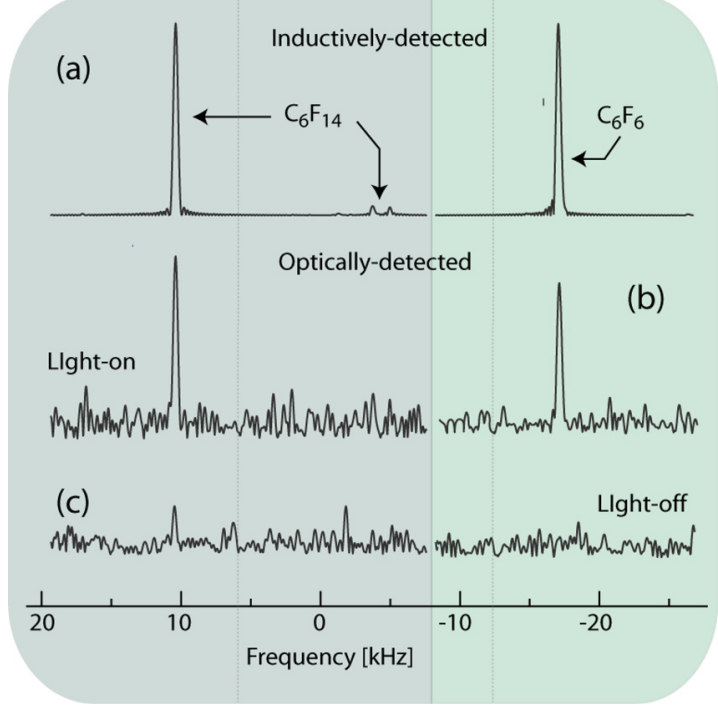

FIG. 4. ODNMR high-resolution spectroscopy. (a) Coil- and (b) optically detected ${ }^{19} \mathrm{~F}$ NMR spectra of perfluorohexane (left) and hexafluorobenzene (right) at $376 \mathrm{MHz}$ after 16 and $8 \times 10^{3}$ repetitions, respectively. Because of the large chemical-shift range, two observations at distinct carrier frequencies (faint dotted lines) were necessary. Note that a direct sensitivity comparison is not warranted here because the coil and the laser beam are effectively probing different sample volumes. See text for details. (c) Same as in (b) but with the beam blocked before the photodetector.

mode) of the $T_{2}$-weighted echo: In Fig. 4(a), the frequency doublet due to inequivalent $\mathrm{CF}_{2}$ fluorines in fluorohexane is barely observable, possibly due to slower tumbling of intrachain nuclei. Optical detection renders a similar pattern [Fig. 4(b)] although the relative amplitude of the aromatic peak is slightly smaller than in the inductive counterpart. As expected, no background signal is observed when the laser beam is blocked [Fig. 4(c)]. On a final note we warn that using the results of Figs. 3 and 4 to establish a comparison between the sensitivities of inductive and OFR detection is inadequate under the present experimental conditions. We will return to this important point later in this paper.

\section{DISCUSSION}

Equation (5) predicts a relationship between the measured Faraday rotation angles and the set of hyperfine coupling constants (hfcc). Provided that the relative number concentrations of nuclear-spins with different chemical-shifts are known, estimates of the hfcc relative amplitude at different molecular sites may be obtained by comparison of the signal intensities in the optically detected NMR spectrum. The data presented in Fig. 4 serve as a case example: Using the inductively detected resonance spectrum as a reference (factoring in number concentration as well as transverse relaxation), we conclude that the hyperfine coupling at ${ }^{19} \mathrm{~F}$ sites in the aromatic and $-\mathrm{CF}_{3}$ groups have similar magnitude. By the same token, the absence of an observable $-\mathrm{CF}_{2}$ resonance in the optically detected spectrum suggests that the corresponding ${ }^{19} \mathrm{~F}$ hfcc can only be similar or smaller. For completeness, we note that these relative values - valid for closed-shell, diamagnetic systems - differ substantially from those found in radical species with the same local structure: Namely, in $-\mathrm{CF}_{2}-\mathrm{CF}_{3}$ radicals ${ }^{13,14}$ the ${ }^{19} \mathrm{~F}$ hfcc at alpha $\left(-\mathrm{CF}_{2}\right)$ sites

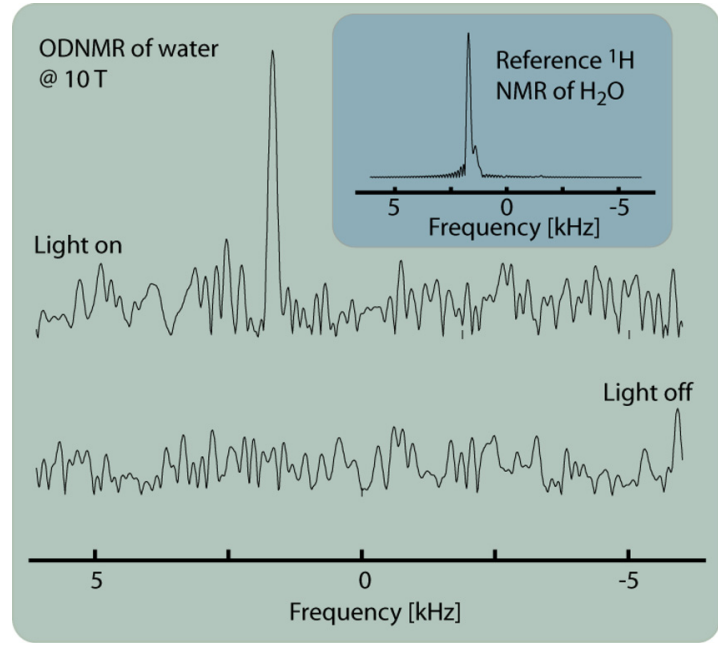

FIG. 5. Inductive (Insert) and OFR-detected (main) NMR of protons in water at $9.4 \mathrm{~T}$ after 16 and $10^{4}$ scans, respectively. Illumination, sampling, and processing conditions are identical to those in Fig. 3. As in all our experiments, no ODNMR signal is present when the light beam is blocked.

$(240 \mathrm{MHz})$ is found to be eight times greater than in beta $\left(-\mathrm{CF}_{3}\right)$ sites; further, Electron Paramagnetic Resonance studies on fluorinated benzenes ${ }^{15}$ set the ${ }^{19} \mathrm{~F}$ hyperfine constant at an intermediate value $(70 \mathrm{MHz})$.

The above considerations can be extended to establish a comparison between the hyperfine constants experienced by other nuclear species. For example, Fig. 5 shows the optically detected ${ }^{1} \mathrm{H}$ NMR spectrum of pure water: Except for the resonance frequency $(400 \mathrm{MHz})$, sampling (and processing) conditions were identical to those used in Fig. 4. In order to take into account differing factors (including nuclear-spin concentration, transverse relaxation time, and spectrometer sensitivity, all greater for protons), we compared the amplitude ratios between the optically and inductively detected signals in $\mathrm{C}_{6} \mathrm{~F}_{6}$ and $\mathrm{H}_{2} \mathrm{O}$. We found that the nuclear susceptibility is approximately three times weaker for water protons, a conclusion in qualitative agreement with the observation that hyperfine couplings tend to grow with higher atomic number. ${ }^{6}$

Ideally, determination of the absolute amplitude of the nuclear-spin-induced Faraday rotation angles - and thus the corresponding nuclear field and hyperfine constants, formula (5) - follows from a system calibration based on (regular) OFR observations in a fluid of known Verdet constant [see formulas (1)-(3)]. In the present case, this task is complicated by the absence of a known standard for the "unusual" conditions of our OFR measurements (i.e., fast modulation of the magnetic field collinear with the beam and strong dc component in the perpendicular direction). More specifically, our data ${ }^{11}$ show that, while the amplitude of the (regular) OFR signal is only marginally sensitive to the $B_{1}$ frequency (which we scanned from $\sim 3$ to $400 \mathrm{MHz}$ ), the addition of a strong dc field $B_{0}$ does lead to a factor of 2-3 reduction, thus introducing an ambiguity. For calibration purposes, we chose to ignore the $B_{0}$ dependence and use the known Verdet constant $V \equiv \theta^{(F)} /\left(L B_{1}\right)$ of water as a reference. With this convention, we determine that the sensitivity of our system is $300 \mathrm{nrad} / \mathrm{Hz}^{1 / 2}$; for ${ }^{19} \mathrm{~F}$, we find $\theta_{N}$ of order $10^{-8} \mathrm{rad}$ and 
the corresponding nuclear field $B_{N}$ in the range $10^{-7} \mathrm{~T}$. The "effective" hyperfine constant $a_{H} /(2 \pi \hbar) \sim g^{*} \mu_{B} B_{N}\left(2 \pi \hbar\left\langle I_{z}\right\rangle\right)$ is found to be of order $\sim 300 \mathrm{MHz}(100 \mathrm{G})$ in ${ }^{19} \mathrm{~F}$ and roughly a third of this value for ${ }^{1} \mathrm{H}$ in water.

When assessing OFR as an alternative detection modality, it is worth highlighting the ability of the present approach to monitor nuclear-spins after spectrometer-timed rf excitation, a feature not found in most optically detected NMR protocols. The latter makes this strategy potentially compatible with the myriad of multipulse sequences that find widespread use in modern NMR: Note in this regard that the train of $\pi$-pulses used in our experiments serves the only purpose of extending the signal duration-with the corresponding SNR improvement-and should be unnecessary in a wellshimmed magnet. Provided the fluid remains transparent over a broad spectral range, additional information could be gained by changing the illumination wavelength (as the OFR signal amplitude is sensitive to the offset relative to a given optical transition).

In its simplest form, OFR-detected NMR suffers from poor sensitivity, a major disadvantage in studies of lowconcentration molecular moieties in solution. Faraday rotation, however, is proportional to the (effective) sample length-not volume-meaning that the SNR observed here should remain virtually unaltered if the sample is confined to a fluid-filled, hollow-core fiber (e.g., a photonic crystal fiber) only 1 or $2 \mathrm{~cm}$ long. ${ }^{16}$ As pointed out in Ref. 6, the combined use of stronger light and a longer, coiled fiber is conceivable and could lead to substantial signal amplification. ${ }^{17}$ Similarly, we note that the overall rotation of a laser beam is doubled if the light, after passing through the medium once, is reflected back through it a second time. This remarkable feature makes the use of "Fabry-Pérot-like" geometries particularly appealing because the expected gain becomes comparable to the cavity finesse. ${ }^{18}$ Preliminary results in our laboratory demonstrate 30 -fold OFR amplification in a low-Q, liquid-filled cavity at zero dc field, ${ }^{19}$ and substantially superior signal gains seem well within reach. This path to higher sensitivity could benefit from recent progress in optofluidics, where centimeter-long optical cavities embedded in a microchip have been exploited to induce lasing of the core fluid in a liquid-liquid fiber $^{20}$ or within a single mode channel waveguide. ${ }^{21}$ For example, for $\sim 300 \mathrm{pl}$ of perfluorohexane within a 5 - $\mu \mathrm{m}$-diameter 1.5 -cm-long channel, one would expect a single-shot SNR of 2 if we double the illumination intensity (from 1.5 to $3 \mathrm{~mW}$ ) and use a cavity of finesse $F \sim 50$ (a 100-fold improvement over the results reported here). In this limit, the resulting sensitivity becomes comparable to that typical of microcoil detection. ${ }^{22}$

On a final note, we point that the use of other cavity geometries - such as that of spherical, disk, or toroidal resonators-poses an intriguing challenge. With diameters ranging from $\sim 100$ to $\sim 30 \mu \mathrm{m}$, ultrahigh quality factors exceeding $10^{9}$ have been demonstrated. ${ }^{23}$ While experimental studies of Faraday rotation in these resonators are lacking, numerical estimates ${ }^{24}$ predict Faraday rotation enhancement of order of $10^{6}-10^{8}$ for optical modes with azimuthal numbers 50-60. Studies that demonstrate the ability of these resonators to probe the composition of a host fluid medium via changes in the index of refraction ${ }^{25}$ could, perhaps, be extended to reconstruct a NMR spectrum. In this same context, we note that rather than probing the medium surrounding the resonator, the sample of interest-shaped in the form of a droplet embedded in an immiscible fluid-could be observed directly, ${ }^{26}$ potentially providing an alternate approach to liquid-state NMR of minuscule sample volumes.

\section{ACKNOWLEDGMENTS}

We are indebted to Linden Langhorne for technical assistance. We thank Professor Jeffrey Reimer, Professor Alexej Jerschow, Professor Dieter Suter, and Dr. Jonathan Hodges for their comments on this manuscript. We acknowledge support from NSF through Grant Nos. CHE-0545461 and CHE-0820416. D.S. acknowledges funding from the European Research Council under the European Community's Seventh Framework Programme: ERC grant Agreement No. 205119.

${ }^{1}$ D. Budker and M. Romalis, Nat. Phys. 3, 227 (2007).

${ }^{2}$ J. M. Kikkawa and D. D. Awschalom, Science 287, 473 (2000).

${ }^{3}$ W. S. Warren, S. Mayr, D. Goswami, and A. P. West, Jr., Science 255, 1683 (1992)

${ }^{4}$ A. D. Buckingham and L. C. Parlett, Science 264, 1748 (1994).

${ }^{5}$ R. A. Harris and I. Tinoco, J. Chem. Phys. 101, 9289 (1994).

${ }^{6}$ I. M. Savukov, S.-K. Lee, and M. V. Romalis, Nature (London) 442 , 1021 (2006).

${ }^{7}$ L. D. Barron, Molecular Light Scattering and Optical Activity (Cambridge University Press, New York, 1982).

${ }^{8}$ A. D. Buckingham and P. J. Stephens, Annu. Rev. Phys. Chem. 17, 399 (1966).

${ }^{9}$ A. Abragam, The Principles of Nuclear Magnetism (Oxford University Press, Oxford, 1970).

${ }^{10}$ C. A. Meriles, Concepts Magn. Reson. 32A, 79 (2008).

${ }^{11}$ See supplementary material at http://dx.doi.org/10.1063/1.3502484 for the materials and methods.

${ }^{12}$ C. P. Slichter, Principles of Magnetic Resonance (Springer, New York, 1996).

${ }^{13}$ P. J. Krusic and R. C. Bingham, J. Am. Chem. Soc. 98, 230 (1976).

${ }^{14}$ J. M. Martell, R. J. Boyd, and L. A. Eriksson, J. Phys. Chem. 99, 623 (1995).

${ }^{15}$ A. R. Rakitin, D. Yff, and C. Trapp, J. Chem. Phys. A 107, 6281 (2003).

${ }^{16}$ F. M. Cox, A. Argyros, and M. C. J. Large, Opt. Express 14, 4135 (2006).

${ }^{17}$ F. Maystre and A. Bertholds, Opt. Lett. 14, 587 (1989).

${ }^{18}$ R. Rosenberg, C. B. Rubinstein, and D. R. Herriott, Appl. Opt. 3, 1079 (1964).

${ }^{19}$ D. Pagliero, Y. Li, S. Fisher, and C. A. Meriles (in preparation).

${ }^{20}$ D. Z. Vezenov, B. T. Mayers, R. S. Conroy, G. M. Whitesides, P. T. Snee, Y. Chan, D. G. Nocera, and M. G. Bawendi, J. Am. Chem. Soc. 127, 8952 (2005).

${ }^{21}$ Z. Li, Z. Zhang, and D. Psaltis, Opt. Express 14, 10494 (2006).

${ }^{22}$ D. L. Olson, T. L. Peck, A. G. Webb, R. L. Magin, and J. V. Sweedler, Science 270, 1967 (1995).

${ }^{23}$ K. J. Vahala, Nature (London) 424, 839 (2003).

${ }^{24}$ L. Deych, V. Menon, C.A. Meriles (in preparation).

${ }^{25}$ A. M. Armani, D. K. Armani, B. Min, and K. Vahala, Appl. Phys. Lett. 87, 151118 (2005).

${ }^{26}$ M. Hossein-Zadeh and K. J. Vahala, Opt. Express 14, 10800 (2006). 